CUBO A Mathematical Journal Vol.16, No $N^{\mathrm{O}}$, (81-93). March 2014

\title{
Characterizations for certain analytic functions by series expansions with Hadamard gaps
}

\author{
A. EL-SAYED AHMED \\ Mathematics Department, \\ Faculty of Science, \\ Sohag University, \\ 82524 Sohag, Egypt \\ ahsayed80@hotmail.com
}

\author{
A. Kamal \\ Department of Mathematics, \\ Faculty of Science, \\ Port Said University, \\ Port Said, Egypt \\ alaa_mohamed10yahoo.com
}

\author{
T.I. YASSEN \\ Department of Mathematics, \\ Faculty of Science, \\ El Azhar University at Assiut, \\ Assiut, Egypt \\ taha_hmour@yahoo.com
}

\begin{abstract}
In this paper we characterize $Q_{\kappa, \omega}(p, q)$ functions by lacunary series under mild conditions posed on the weight functions $K$ and $\omega$, where $Q_{K, \omega}(p, q)$ is a space of analytic functions defined in the unit disk generalizing the well known analytic Besov-type space.

\section{RESUMEN}

En este artículo caracterizamos las funciones $Q_{K, \omega}(p, q)$ por series lacunarias bajo condiciones medianas impuestas en las funciones de peso $K$ y $\omega$, donde $Q_{K, \omega}(p, q)$ es un espacio de funciones analíticas definidas en el disco unitario generalizando el conocido espacio analítico del tipo Besov.
\end{abstract}

Keywords and Phrases: $Q_{\kappa, \omega}(p, q)$-type spaces, Lacunary series.

2010 AMS Mathematics Subject Classification: 30B10, 30B50, 46E15. 


\section{Introduction}

Let $\mathbb{D}=\{z \in \mathbb{C}:|z|<1\}$ be the open unit disk in the complex plane $\mathbb{C}$, let $\mathrm{H}(\mathbb{D})$ denote the class of functions analytic in the unit disc $\mathbb{D}$, while $d A(z)$ denotes the Lebesgue area measure on the plane, normalized so that $A(\mathbb{D})=1$.

Let the Green's function of $\mathbb{D}$ be defined as $g(z, a)=\log \frac{1}{\varphi_{a}(z)}$, where $\varphi_{a}(z)=\frac{a-z}{1-\bar{a} z}$ is the Möbius transformation related to the point $a \in \mathbb{D}$. For $0<r<1$, let $\mathbb{D}(a, r)=\left\{r \in \mathbb{D}:\left|\varphi_{a}(z)\right|<r\right\}$ be the pseudo-hyperbolic disk with center $a \in \mathbb{D}$ and radius $r$.

Definition 1.1. 177 Let $\mathrm{K}:[0, \infty) \rightarrow[0, \infty)$ be right-continuous and nondecreasing function, $0<\mathrm{p}<\infty,-2<\mathrm{q}<\infty$ and for given reasonable function $\omega:(0,1] \rightarrow(0, \infty)$, an analytic function $\mathrm{f}$ in $\mathbb{D}$ is said to belong to the space $\mathrm{Q}_{\mathrm{K}, \omega}(\mathrm{p}, \mathrm{q})$ if

$$
\|f\|_{Q_{K, \omega(p, q)}}=\sup _{a \in \mathbb{D}} \int_{\mathbb{D}}\left|f^{\prime}(z)\right|^{p} \frac{(1-|z|)^{q}}{\omega^{\mathfrak{p}}(1-|z|)} K(g(z, a)) d A(z)<\infty .
$$

In the past few decades both Taylor and Fourier series expansions for various classes of analytic function spaces where the studies are done by the help of Hadamard gap class (see [1, 3, 10, 16] and others).

It is well known that a lacunary series belongs to BMOA if and only if it is in the Hardy space $\mathrm{H}^{2}$, (see [2]) for example. Very recently, in [6, 17, 14, there are some characterizations for some classes of meromorphic functions by the coefficients of certain lacunary series expansions in the unit disk. On the other hand there are some studies of the same problem in Clifford analysis (see [4, 15, 12, 13).

We assume throughout the paper that

$$
\int_{0}^{1}(1-r)^{\mathrm{q}} \frac{K\left(\log \frac{1}{r}\right)}{\omega^{\mathfrak{p}}\left(\log \frac{1}{r}\right)} r d r<\infty .
$$

An important tool in the study of $Q_{K, \omega}(p, q)$ space is the auxiliary functions $\phi_{K}$ and $\psi_{\omega}$ defined by

$$
\phi_{K}(s)=\sup _{0<t<1} \frac{K(s t)}{K(t)}, \quad 0<s<\infty
$$

and

$$
\psi_{\omega}(s)=\sup _{0<t<1} \frac{\omega(s t)}{\omega(t)}, \quad 0<s<1 .
$$

The following conditions have played a crucial roles in the study of $Q_{K, \omega}(p, q)$ space:

$$
\int_{1}^{\infty} \phi_{K}(s) \frac{d s}{s^{2}}<\infty
$$

(See [9, 17]) for example.

$$
\int_{1}^{\frac{1}{t}} \psi_{\omega}(s) \frac{d s}{s^{2}}<\infty
$$


The function theory of $\mathrm{Q}_{\kappa, \omega}(\mathrm{p}, \mathrm{q})$ obviously depends on the properties of $\mathrm{K}$ and $\omega$. Given the weight functions $\omega_{1}, \omega_{2}, K_{1}$ and $K_{2}$. We write $\omega_{1} \lesssim \omega_{2}, K_{1} \lesssim K_{2}$ if there exists a constant $C>0$, independent of $t$, such that $\omega_{1}(t) \leq C \omega_{2}(t), K_{1}(t) \leq C K_{2}(t)$ for all $t$. The notation $\omega_{1} \gtrsim \omega_{2}, K_{1} \gtrsim K_{2}$ is used in a similar fashion. When $\omega_{1} \lesssim \omega_{2} \lesssim \omega_{1}$, we write $\omega_{1} \approx \omega_{2}$. Also for $\mathrm{K}_{1} \lesssim \mathrm{K}_{2} \lesssim \mathrm{K}_{1}$, we write $\mathrm{K}_{1} \approx \mathrm{K}_{2}$.

\section{Auxiliary Lemmas}

In what follows we say $f \lesssim g$ (for two functions $f$ and $g$ ) if there is a constant $\mathrm{C}$ such that $\mathrm{f} \leq \mathrm{Cg}$. We say $f \approx g$ (that is, $f$ is comparable with $g$ ) whenever $g \lesssim f \lesssim g$. In this section we prove several result about the weight function that are needed for subsequent sections and are of some independent interest.

Lemma 2.1. 20] If $\mathrm{K}$ satisfies condition (11), then the function

$$
\mathrm{K}^{*}(\mathrm{t})=\mathrm{t} \int_{\mathrm{t}}^{\infty} \frac{\mathrm{K}(\mathrm{s})}{\mathrm{s}^{2}} \mathrm{ds} \quad(\text { where, } 0<\mathrm{t}<\infty)
$$

has the following properties :

(A) $\mathrm{K}^{*}$ is nondecreasing on $(0, \infty)$.

(B) $\mathrm{K}^{*}(\mathrm{t}) / \mathrm{t}$ is nondecreasing on $(0, \infty)$.

(C) $\mathrm{K}^{*}(\mathrm{t}) \geq \mathrm{K}(\mathrm{t})$ for all $\mathrm{t} \in(0, \infty)$.

(D) $\mathrm{K}^{*} \lesssim \mathrm{K}$ on $(0,1]$.

If $\mathrm{K}(\mathrm{t})=\mathrm{K}(1)$ for $\mathrm{t} \geq 1$, then we also have

(E) $\mathrm{K}^{*}(\mathrm{t})=\mathrm{K}^{*}(1)=\mathrm{K}(1)$ for $\mathrm{t} \geq 1$, so $\mathrm{K}^{*} \approx \mathrm{K}$ on $(0, \infty)$.

Lemma 2.2. [20] If $\mathrm{K}$ satisfies condition (1), then we can find another non-negative weight function given by

$$
\mathrm{K}^{*}(\mathrm{t})=\mathrm{t} \int_{\mathrm{t}}^{\infty} \frac{\mathrm{K}(\mathrm{s})}{\mathrm{s}^{2}} \mathrm{ds} \quad(\text { where, } 0<\mathrm{t}<\infty),
$$

such that $\mathrm{Q}_{\mathrm{K}, \omega}(\mathrm{p}, \mathrm{q})=\mathrm{Q}_{\mathrm{K}^{*}, \omega}(\mathrm{p}, \mathrm{q})$ and that the new function $\mathrm{K}^{*}$ has the following properties :

(A) $\mathrm{K}^{*}$ is nondecreasing on $(0, \infty)$.

(B) $\mathrm{K}^{*}(\mathrm{t}) / \mathrm{t}$ is nondecreasing on $(0, \infty)$. 
(c) $\mathrm{K}^{*}(\mathrm{t})$ satisfies condition (1)

(d) $\mathrm{K}^{*}(2 \mathrm{t}) \approx \mathrm{K}^{*}(\mathrm{t})$ on $(0, \infty)$.

(e) $\mathrm{K}^{*}(\mathrm{t}) \approx \mathrm{K}(\mathrm{t})$ on $(0,1]$.

(f) $\mathrm{K}^{*}$ is differentiable on $(0, \infty)$.

(g) $\mathrm{K}^{*}$ is concave on $(0, \infty)$.

(h) $\mathrm{K}^{*}(\mathrm{t})=\mathrm{K}^{*}(1)$ for $\mathrm{t} \geq 1$,

Lemma 2.3. [8] If $\omega$ satisfies condition [2), then the function

$$
\omega^{*}(t)=t \int_{t}^{1} \frac{\omega(s)}{s^{2}} d s \quad(\text { where, } 0<t<1),
$$

has the following properties :

(A) $\omega^{*}$ is nondecreasing on $(0,1)$.

(B) $\omega^{*}(\mathrm{t}) / \mathrm{t}$ is nondecreasing on $(0,1)$.

(C) $\omega^{*}(\mathrm{t}) \geq \omega(\mathrm{t})$ for all $\mathrm{t} \in(0,1)$.

(D) $\omega^{*} \lesssim \omega$ on $(0,1)$.

If $\omega(\mathrm{t})=\omega(1)$ for $\mathrm{t} \geq 1$, then we also have

(E) $\omega^{*}(t)=\omega^{*}(1)=\omega(1)$ for $t \geq 1$, so $\omega^{*} \approx \omega$ on $(0,1)$.

Lemma 2.4. Let $\alpha \geq 1$ and $\beta>0$. If $\mathrm{K}$ satisfies (1) and $\omega$ satisfies (2), then

$$
\int_{0}^{1} r^{\alpha-1}\left(\log \frac{1}{r}\right)^{\beta-1} \frac{K\left(\log \frac{1}{r}\right)}{\omega^{p}\left(\log \frac{1}{r}\right)} d r \approx\left(\frac{1}{\alpha}\right)^{\beta} \frac{K\left(\frac{1}{\alpha}\right)}{\omega^{p}\left(\frac{1}{\alpha}\right)}
$$

Proof. Let

$$
I=\int_{0}^{1} r^{\alpha-1}\left(\log \frac{1}{r}\right)^{\beta-1} \frac{K\left(\log \frac{1}{r}\right)}{\omega^{p}\left(\log \frac{1}{r}\right)} d r .
$$

By the change variables we have

$$
I=\int_{0}^{1} e^{-\alpha t} t^{\beta-1} \frac{K(t)}{\omega^{p}(t)} d t
$$


We write $\mathrm{I}=\mathrm{I}_{1}+\mathrm{I}_{2}$ where

$$
I_{1}=\int_{\frac{1}{\alpha}}^{1} e^{-\alpha t} t^{\beta-1} \frac{K(t)}{\omega^{p}(t)} d t
$$

and

$$
I_{2}=\int_{0}^{\frac{1}{\alpha}} e^{-\alpha t} t^{\beta-1} \frac{K(t)}{\omega^{p}(t)} d t .
$$

By Lemma 2.1 and Lemma 2.2, we have

$$
\begin{aligned}
& I_{1} \leq \frac{K\left(\frac{1}{\alpha}\right)}{\left(\frac{1}{\alpha}\right)} \times \frac{\left(\frac{1}{\alpha}\right)}{\omega^{\mathcal{p}\left(\frac{1}{\alpha}\right)}} \int_{\frac{1}{\alpha}}^{1} e^{-\alpha t} t^{\beta-1} d t \\
& =\frac{K\left(\frac{1}{\alpha}\right)}{\omega^{p}\left(\frac{1}{\alpha}\right)} \int_{\frac{1}{\alpha}}^{1} e^{-\alpha t} t^{\beta-1} d t .
\end{aligned}
$$

Making the change of variables $s=\alpha t$, we have

$$
\begin{aligned}
& I_{1} \leq \frac{K\left(\frac{1}{\alpha}\right)}{\omega^{\mathcal{p}}\left(\frac{1}{\alpha}\right)} \int_{1}^{\alpha} e^{-s} s^{\beta-1}\left(\frac{1}{\alpha}\right)^{\beta-1}\left(\frac{1}{\alpha}\right) d s \\
& =\frac{K\left(\frac{1}{\alpha}\right)}{\omega^{\mathcal{p}}\left(\frac{1}{\alpha}\right)}\left(\frac{1}{\alpha}\right)^{\beta} \int_{1}^{\alpha} e^{-s} s^{\beta-1} d s .
\end{aligned}
$$

Then

$$
\mathrm{I}_{1} \leq \mathrm{C}(\beta) \frac{\mathrm{K}\left(\frac{1}{\alpha}\right)}{\omega^{\mathfrak{p}}\left(\frac{1}{\alpha}\right)}\left(\frac{1}{\alpha}\right)^{\beta} .
$$

Since $K(t)$ and $\omega(t)$ are non-decreasing, then by making the change of variables $s=\alpha t$, we obtain

$$
\mathrm{I}_{2} \leq \frac{\mathrm{K}\left(\frac{1}{\alpha}\right)}{\omega^{\mathrm{p}}\left(\frac{1}{\alpha}\right)} \int_{0}^{\frac{1}{\alpha}} e^{-\alpha \mathrm{t}} \mathrm{t}^{\beta-1} d \mathrm{t}
$$

and

$$
\mathrm{I}_{2} \leq \frac{\mathrm{K}\left(\frac{1}{\alpha}\right)}{\omega^{\mathcal{P}}\left(\frac{1}{\alpha}\right)}\left(\frac{1}{\alpha}\right)^{\beta} \int_{0}^{1} e^{-s} s^{\beta-1} \text { ds. }
$$

Then

$$
\mathrm{I}_{2} \leq \mathrm{C}(\beta) \frac{\mathrm{K}\left(\frac{1}{\alpha}\right)}{\omega^{\mathfrak{p}}\left(\frac{1}{\alpha}\right)}\left(\frac{1}{\alpha}\right)^{\beta}
$$

Combining this with what was proved in the previous paragraph, we have

$$
\int_{0}^{1} r^{\alpha-1}\left(\log \frac{1}{r}\right)^{\beta-1} \frac{K\left(\log \frac{1}{r}\right)}{\omega^{p}\left(\log \frac{1}{r}\right)} d r \leq C(\beta) \frac{K\left(\frac{1}{\alpha}\right)}{\omega^{p}\left(\frac{1}{\alpha}\right)}\left(\frac{1}{\alpha}\right)^{\beta},
$$

where $C(\beta)$ is constant which only depends on $(\beta)$.

On the other hand, recall that $\mathrm{K}(\mathrm{t})$ and $\omega(\mathrm{t})$ are non-decreasing. Then,

$$
I \geq \int_{\frac{1}{\alpha}}^{1} e^{-\alpha t} t^{\beta-1} \frac{K(t)}{\omega^{\mathcal{p}}(t)} d t \geq \frac{K\left(\frac{1}{\alpha}\right)}{\omega^{\mathcal{P}}\left(\frac{1}{\alpha}\right)} \int_{\frac{1}{\alpha}}^{1} e^{-\alpha t} t^{\beta-1} d t .
$$


Making the change of variables $s=\alpha t$, we have

$$
I \geq C(\beta) \frac{K\left(\frac{1}{\alpha}\right)}{\omega^{p}\left(\frac{1}{\alpha}\right)}\left(\frac{1}{\alpha}\right)^{\beta}
$$

Then

$$
\int_{0}^{1} r^{\alpha-1}\left(\log \frac{1}{r}\right)^{\beta-1} \frac{K\left(\log \frac{1}{r}\right)}{\omega^{p}\left(\log \frac{1}{r}\right)} d r \approx C(\beta) \frac{K\left(\frac{1}{\alpha}\right)}{\omega^{p}\left(\frac{1}{\alpha}\right)}\left(\frac{1}{\alpha}\right)^{\beta} .
$$

This completes the proof of the lemma.

Lemma 2.5. Let $0<\gamma \leq 1$ and $\eta(r)=\sum_{n=0}^{\infty} 2^{n \gamma} r^{2^{n}}, 0 \leq r<1$, then

$$
\eta(r) \leq 2 \Gamma(\gamma)\left(\log \frac{1}{r}\right)^{-\gamma}
$$

Then above result can be found in [15].

Lemma 2.6. [19]

$$
\frac{1}{2 \pi} \int_{0}^{2 \pi} g\left(r e^{i \theta}, a\right) d \theta= \begin{cases}\log \frac{1}{|a|}, & 0<r \leq|a|, \\ \log \frac{1}{r}, & |a|<r \leq 1 .\end{cases}
$$

By Jensen's formula(see [11, [18]), we can directly obtain the above result.

Theorem 2.7. Let K satisfy condition (1) and $\omega$ satisfy condition (2). Suppose that

$$
f(x)=\sum_{n=1}^{\infty} a_{n} x^{n}
$$

with $\mathrm{a}_{\mathrm{n}} \geq 0$. For $\alpha>0, \mathrm{p}>0$, we have that

$$
\int_{0}^{1}(1-x)^{\alpha-1}(f(x))^{p} \frac{K\left(\log \frac{1}{x}\right)}{\omega^{p}\left(\log \frac{1}{x}\right)} d x \approx \sum_{n=0}^{\infty} 2^{-n \alpha} t_{n}^{p} \frac{K\left(\frac{1}{2^{n}}\right)}{\omega^{p}\left(\frac{1}{2^{n}}\right)}
$$

where $\mathrm{t}_{\mathrm{n}}=\sum_{\mathrm{k} \in \mathrm{I}_{\mathrm{n}}} \mathrm{a}_{\mathrm{k}}, \mathrm{n} \in \mathbb{N}, \mathrm{I}_{\mathrm{n}}=\left\{\mathrm{k}: 2^{\mathrm{n}} \leq \mathrm{k}<2^{\mathrm{n}+1} ; \mathrm{k} \in \mathbb{N}\right\}$.

Proof. Let $r_{n}=1-2^{-n}, n=1,2, \ldots$, then $r_{n}^{2^{n}-1} \geq \frac{1}{e}$. By Lemma 2.3. we have that

$$
\begin{aligned}
& \int_{0}^{1}\left(\sum_{n=1}^{\infty} a_{n} r^{n}\right)^{p}(1-r)^{\alpha-1} \frac{K\left(\log \frac{1}{r}\right)}{\omega^{p}\left(\log \frac{1}{r}\right)} d r \\
& \geq \int_{\frac{1}{2}}^{1}\left(\sum_{n=0}^{\infty} t_{n} r^{2^{n+1}-1}\right)^{p}(1-r)^{\alpha-1} \frac{K\left(\log \frac{1}{r}\right)}{\omega^{p}\left(\log \frac{1}{r}\right)} d r \\
& \gtrsim \sum_{n=0}^{\infty} t_{n}^{p}\left(r_{n+1}^{2^{n+1}-1}\right)^{p} \int_{r_{n+1}}^{r_{n+2}}(1-r)^{\alpha-1} \frac{K\left(\log \frac{1}{r_{n+1}}\right)}{\omega^{p}\left(\log \frac{1}{r_{n+1}}\right)} d r \\
& \gtrsim \sum_{n=0}^{\infty} 2^{-n \alpha} t_{n}^{p} \frac{K\left(\frac{1}{2^{n}}\right)}{\omega^{p}\left(\frac{1}{2^{n}}\right)} .
\end{aligned}
$$


The last inequality holds because of $\left(\log \frac{1}{r}\right) \geq 1-r$.

Conversely, we first suppose that $p>1$. Let $\gamma=\min \{1, \alpha / p\}$ and $\eta(r)=\sum_{k=0}^{\infty} 2^{k \gamma} r^{2 k}$, $0 \leq r<1$. Then by Jensen's inequality (see [11, 18]), we have

$$
\left(\sum_{n=0}^{\infty} a_{n} r^{n}\right)^{p} \leq\left(\sum_{n=0}^{\infty} t_{n} r^{2^{n}}\right)^{p} \lesssim(\eta(r))^{p-1} \sum_{n=0}^{\infty} 2^{n \gamma(1-p)} r^{2 n}\left|t_{n}\right|^{p}
$$

From Lemma 2.2, Lemma 2.3, Lemma 2.4 and Lemma 2.5, it follows that

$$
\begin{aligned}
& \int_{0}^{1}\left(\sum_{n=1}^{\infty} a_{n} r^{n}\right)^{p}(1-r)^{\alpha-1} \frac{K\left(\log \frac{1}{r}\right)}{\omega^{p}\left(\log \frac{1}{r}\right)} d r \\
& \leq \int_{0}^{1}(2 \Gamma(\gamma))^{p-1}\left(\log \frac{1}{r}\right)^{-\gamma(p-1)}\left(\sum_{n=0}^{\infty} 2^{n \gamma(1-p)} r^{2 n}\left|t_{n}\right|^{p}(1-r)^{\alpha-1}\right) \frac{K\left(\log \frac{1}{r}\right)}{\omega^{p}\left(\log \frac{1}{r}\right)} d r \\
& \leq 2^{n \gamma(1-p)} \sum_{n=0}^{\infty}\left|t_{n}\right|^{p} \int_{0}^{1} r^{2 n-1}\left(\log \frac{1}{r}\right)^{-\gamma(p-1)+\alpha-1} \frac{K\left(\log \frac{1}{r}\right)}{\omega^{p}\left(\log \frac{1}{r}\right)} d r \\
& \leq 2^{n \gamma(1-p)} \sum_{n=0}^{\infty}\left|t_{n}\right|^{p}\left(\frac{1}{2^{n}}\right)^{-\gamma(p-1)+\alpha} \frac{K\left(\log \frac{1}{r}\right)}{\omega^{p}\left(\log \frac{1}{r}\right)} \\
& \leq \sum_{n=0}^{\infty} 2^{-n \alpha} t_{n}^{p} \frac{K\left(\frac{1}{2^{n}}\right)}{\omega^{p}\left(\frac{1}{2^{n}}\right)}
\end{aligned}
$$

Denote $r(1-r)^{\alpha-1} \leq\left(\log \frac{1}{r}\right)^{\alpha-1}, \quad 0 \leq r<1$. Secondly suppose that $p=1$, by Lemma 2.4 we obtain that

$$
\begin{aligned}
& \int_{0}^{1}\left(\sum_{n=1}^{\infty} a_{n} r^{n}\right)(1-r)^{\alpha-1} \frac{K\left(\log \frac{1}{r}\right)}{\omega^{p}\left(\log \frac{1}{r}\right)} d r \\
& \int_{0}^{1}\left(\sum_{n=0}^{\infty} t_{n} r^{2^{n}}\right)(1-r)^{\alpha-1} \frac{K\left(\log \frac{1}{r}\right)}{\omega^{p}\left(\log \frac{1}{r}\right)} d r \\
& \leq \sum_{n=0}^{\infty} t_{n} \int_{0}^{1}\left(r^{2^{n}-1}\right) r(1-r)^{\alpha-1} \frac{K\left(\log \frac{1}{r}\right)}{\omega^{p}\left(\log \frac{1}{r}\right)} d r \\
& \leq \sum_{n=0}^{\infty} t_{n} \int_{0}^{1}\left(r^{2^{n}-1}\right)\left(\log \frac{1}{r}\right)^{\alpha-1} \frac{K\left(\log \frac{1}{r}\right)}{\omega^{p}\left(\log \frac{1}{r}\right)} d r \\
& \leq \sum_{n=0}^{\infty} 2^{-n \alpha} t_{n} \frac{K\left(\frac{1}{2^{n}}\right)}{\omega^{p}\left(\frac{1}{2^{n}}\right)} .
\end{aligned}
$$


Finally, if $p<1$, we have

$$
\begin{aligned}
& \int_{0}^{1}\left(\sum_{n=1}^{\infty} a_{n} r^{n}\right)^{p}(1-r)^{\alpha-1} \frac{K\left(\log \frac{1}{r}\right)}{\omega^{p}\left(\log \frac{1}{r}\right)} d r \\
& \int_{0}^{1}\left(\sum_{n=0}^{\infty} t_{n} r^{2^{n}}\right)^{p}(1-r)^{\alpha-1} \frac{K\left(\log \frac{1}{r}\right)}{\omega^{p}\left(\log \frac{1}{r}\right)} d r \\
& \leq \sum_{n=0}^{\infty} t_{n}^{p} \int_{0}^{1}\left(r^{p 2^{n}-1}\right) r(1-r)^{\alpha-1} \frac{K\left(\log \frac{1}{r}\right)}{\omega^{p}\left(\log \frac{1}{r}\right)} d r \\
& \leq \sum_{n=0}^{\infty} t_{n}^{p} \int_{0}^{1}\left(r^{p 2^{n}-1}\right)\left(\log \frac{1}{r}\right)^{\alpha-1} \frac{K\left(\log \frac{1}{r}\right)}{\omega^{p}\left(\log \frac{1}{r}\right)} d r \\
& \leq \sum_{n=0}^{\infty} 2^{-n \alpha} t_{n}^{p} \frac{K\left(\frac{1}{2^{n}}\right)}{\omega^{p}\left(\frac{1}{2^{n}}\right)}
\end{aligned}
$$

From (4), (5) and (6), we obtain the desired result and the proof is therefore established.

\section{$3 \quad$ Main results}

We prove that an analytic function $f$ on the unit disk $\mathbb{D}$ with Hadamard gaps, that is, $f(z)=$ $\sum_{n=1}^{\infty} a_{n} z^{n}$ satisfying $\frac{n_{k+1}}{n_{k}} \geq \lambda>1$ for all $k \in \mathbb{N}$, belongs to the space $Q_{k, \omega}(p, q)$ with mild conditions on the weight functions $K$ and $\omega$ if and only if

$$
\sum_{k=0}^{\infty} n_{k}^{p-q-1}\left|a_{k}\right|^{p} \frac{K\left(\frac{1}{n_{k}}\right)}{\omega^{p}\left(\frac{1}{n_{k}}\right)}<\infty
$$

where $0<p<\infty$ and $-1<q<\infty$. Now, we give the following result:

Theorem 3.1. Let K satisfy condition (1) and $w$ satisfy condition (2). Suppose that

$$
f(z)=\sum_{n=1}^{\infty} a_{n} z^{n}
$$

with $\mathrm{a}_{\mathrm{n}} \geq 0$. For $\alpha>0, \mathrm{p}>0$, we have

$$
\int_{\mathbb{D}}\left|f^{\prime}(z)\right|^{p} \frac{(1-|z|)^{q}}{\omega^{p}(1-|z|)} K\left(\log \frac{1}{|z|}\right) d A(z) \approx \sum_{n=1}^{\infty} n^{p-q-1}\left|a_{n}\right|^{p} \frac{K\left(\frac{1}{n}\right)}{\omega^{p}\left(\frac{1}{n}\right)},
$$

where $\mathrm{t}_{\mathrm{n}}=\sum_{k \in \mathrm{I}_{\mathrm{n}}} \mathrm{a}_{\mathrm{k}}, \quad \mathrm{n} \in \mathbb{N}, \mathrm{I}_{\mathrm{n}}=\left\{\mathrm{k}: 2^{\mathrm{n}} \leq \mathrm{k}<2^{\mathrm{n}+1} ; \mathrm{k} \in \in \mathbb{N}\right\}$

Proof. We write

$$
\mathrm{I}(\mathrm{f})=\int_{\mathbb{D}}\left|f^{\prime}(z)\right|^{\mathrm{p}} \frac{(1-|z|)^{\mathrm{q}}}{\omega^{\mathrm{p}}(1-|z|)} \mathrm{K}\left(\log \frac{1}{|z|}\right) \mathrm{d} A(z) .
$$


Integrating in polar coordinates we gets

by theorem 2.7, we obtain

$$
\begin{aligned}
I(f) & =\int_{0}^{1} \int_{0}^{2 \pi}\left(\sum_{n=1}^{\infty} n\left|a_{n}\right| r^{n-1}\right)^{p}(1-r)^{q} \frac{K\left(\log \frac{1}{r}\right)}{\omega^{p}\left(\log \frac{1}{r}\right)} r d r d \theta \\
& \lesssim \int_{0}^{1}\left(\sum_{n=1}^{\infty} n\left|a_{n}\right| r^{n-1}\right)^{p}(1-r)^{q} \frac{K\left(\log \frac{1}{r}\right)}{\omega^{p}\left(\log \frac{1}{r}\right)} d r
\end{aligned}
$$

$$
\begin{aligned}
& I(f) \lesssim \sum_{n=0}^{\infty} 2^{-n(q+1)} t_{n}^{p} \frac{K\left(\frac{1}{2^{n}}\right)}{\omega^{p}\left(\frac{1}{2^{n}}\right)} \\
& \approx \sum_{n=1}^{\infty} n^{p-q-1}\left|a_{n}\right|^{p} \frac{K\left(\frac{1}{n}\right)}{\omega^{p}\left(\frac{1}{n}\right)} .
\end{aligned}
$$

The proof is therefore established.

Theorem 3.2. Let $0<\mathrm{p}<\infty,-1<\mathrm{q}<\infty$. Let $\mathrm{K}$ satisfy condition (1) and $\omega$ satisfy condition (2). Suppose that

$$
f(z)=\sum_{k=1}^{\infty} a_{k} z^{n_{k}},
$$

has Hadamard gaps, then $\mathrm{f}$ belongs to $\mathrm{Q}_{\mathrm{K}, \omega}(\mathrm{p}, \mathrm{q})$ if and only if

$$
\sum_{k=1}^{\infty} n_{k}^{p-q-1}\left|a_{k}\right|^{p} \frac{K\left(\frac{1}{n_{k}}\right)}{\omega^{p}\left(\frac{1}{n_{k}}\right)}<\infty .
$$

Proof. Suppose that $f(z)=\sum_{k=1}^{\infty} a_{k} z^{n_{k}}$ is a lacunary series in $Q_{k, \omega}(p, q)$. Without loss generality, we assume that $n_{k} \geq 1$. If $f$ has Hadamard gaps, then $M_{p}\left(r, f^{\prime}\right) \approx M_{2}\left(r, f^{\prime}\right)$ (see [21]), where

$$
M_{p}^{p}\left(r, f^{\prime}\right)=\frac{1}{2 \pi} \int_{0}^{2 \pi}\left|f^{\prime}\left(r e^{i} \theta\right)\right|^{p} d \theta
$$

since $f \in Q_{K, \omega}(p, q)$, by theorem 2.7 we obtain

$$
\begin{aligned}
& \infty>\int_{\mathbb{D}}\left|f^{\prime}(z)\right|^{p} \frac{(1-|z|)^{\mathfrak{q}}}{\omega^{\mathfrak{p}}(1-|z|)} K\left(\log \frac{1}{|z|}\right) \mathrm{d} A(z) \\
& =\int_{0}^{1} M_{p}^{p}\left(r, f^{\prime}\right)(1-r)^{q} \frac{K\left(\log \frac{1}{r}\right)}{\omega^{p}\left(\log \frac{1}{r}\right)} r d r, \\
& \gtrsim \int_{0}^{1}\left(\sum_{k=1}^{\infty} n_{k}^{2}\left|a_{k}\right|^{2} r^{2\left(n_{k}-1\right)}\right)^{\frac{p}{2}}(1-r)^{q} \frac{K\left(\log \frac{1}{r}\right)}{\omega^{p}\left(\log \frac{1}{r}\right)} r d r, \\
& \gtrsim \sum_{k=1}^{\infty} 2^{-k(q+1)} t_{k}^{\frac{p}{2}} \frac{K\left(\frac{1}{2^{k}}\right)}{\omega^{p}\left(\frac{1}{2^{k}}\right)}
\end{aligned}
$$

where $t_{k}=\sum_{n_{j} \in I_{k}} n_{j}^{2}\left|a_{j}\right|^{2}$. The Taylor series of $f$ has at most $\left[\log _{\lambda} 2\right]+1$ terms $a_{j} z^{n_{j}}$ when $n_{j} \in I_{k}$ for $k \geq 1$. By Hölder's inequality, we note that $t_{k}^{\frac{p}{2}} \gtrsim \sum_{n_{j} \in I_{k}} n_{j}^{p}\left|a_{j}\right|^{p}$. Then 


$$
\sum_{k=0}^{\infty} n_{k}^{p-q-1}\left|a_{k}\right|^{p} \frac{K\left(\frac{1}{n_{k}}\right)}{\omega^{p}\left(\frac{1}{n_{k}}\right)} \lesssim \sum_{k=0}^{\infty} 2^{-k(q+1)}\left(\sum_{n_{j} \in I_{k}} n_{j}^{p}\left|a_{j}\right|^{p}\right) \frac{K\left(\frac{1}{2^{k}}\right)}{\omega^{p}\left(\frac{1}{2^{k}}\right)}<\infty .
$$

Next suppose that condition (8) holds, we write $z=r e^{i \theta}$ in polar form and observe that

$$
|f(z)|=\sum_{k=1}^{\infty}\left|a_{k}\right| r^{n_{k}}
$$

Since $K$ and $\omega$ satisfies conditions (1) and (2) respectively, $K$ is concave. Then, by Jensen's inequality, Lemma 2.2 and Lemma 2.6, we deduce that

$$
\int_{0}^{2 \pi} K\left(g\left(r e^{i \theta}, a\right)\right) d \theta \leq K\left(\int_{0}^{2 \pi}\left(g\left(r e^{i \theta}, a\right)\right) d \theta\right) \leq K\left(\log \frac{1}{r}\right)
$$

Hence,

$$
\begin{aligned}
& \|f\|_{\mathrm{K}, \omega}^{\mathrm{p}}=\sup _{\mathrm{a} \in \mathbb{D}} \int_{\mathbb{D}}\left|f^{\prime}(z)\right|^{p} \frac{(1-|z|)^{\mathrm{q}}}{\omega^{\mathrm{p}}(1-|z|)} \mathrm{K}\left(\log \frac{1}{|z|}\right) \mathrm{dA}(z) \\
& \leq \sup _{\mathrm{a} \in \mathbb{D}} \int_{0}^{1}\left(\sum_{k=1}^{\infty} n_{k}\left|a_{k}\right| r^{\left(n_{k}-1\right)}\right)^{\mathrm{p}} \frac{(1-r)^{\mathrm{q}}}{\omega^{\mathrm{p}(1-r)}} \\
& \times\left\{\frac{1}{2 \pi} \int_{0}^{2 \pi} K\left(g\left(r e^{i \theta}, a\right)\right) d \theta\right\} r d r \\
& \leq \sup _{a \in \mathbb{D}} \int_{0}^{1}\left(\sum_{k=1}^{\infty} n_{k}\left|a_{k}\right| r^{\left(n_{k}-1\right)}\right)^{p} \frac{(1-r)^{q}}{\omega^{p}(1-r)} K\left(\log \frac{1}{r}\right) r d r
\end{aligned}
$$

by Theorem 2.7, we obtain

$$
\begin{aligned}
\|f\|_{Q_{k, \omega}(p, q)} & \lesssim \sum_{n=0}^{\infty} 2^{-n(q+1)} t_{n}^{p} \frac{K\left(\frac{1}{2^{n}}\right)}{\omega^{p}\left(\frac{1}{2^{n}}\right)} \\
& \lesssim \sum_{K=1}^{\infty} n_{k}^{p-q-1}\left|a_{k}\right|^{p} \frac{K\left(\frac{1}{n_{k}}\right)}{\omega^{p}\left(\frac{1}{n_{k}}\right)}<\infty
\end{aligned}
$$

The proof is therefore established.

Theorem 3.3. Let $0<\mathrm{p}<\infty,-1<\mathrm{q}<\infty$. Let $\mathrm{K}$ satisfy condition (1) and $\mathrm{\omega}$ satisfy condition (2). Suppose that

$$
f(z)=\sum_{k=1}^{\infty} a_{k} z^{n_{k}}
$$

has Hadamard gaps, $\omega>0$, then $\mathrm{f}$ belongs to $\mathrm{Q}_{\kappa, \omega}(\mathrm{p}, \mathrm{q})$ if and only if $\mathrm{f} \in \mathrm{Q}_{\kappa, \omega, \mathrm{o}}(\mathrm{p}, \mathrm{q})$.

Proof. Since sufficiency is obvious because of $\mathrm{Q}_{\kappa, \omega, 0}(\mathrm{p}, \mathrm{q}) \subset \mathrm{Q}_{\kappa, \omega}(\mathrm{p}, \mathrm{q})$.

Now we will prove necessity of Theorem 3.3 Suppose that the lacunary series $f$ belongs to $\mathrm{Q}_{\kappa, \omega}(\mathrm{p}, \mathrm{q})$. We must show that $\mathrm{I}(\mathrm{a}) \rightarrow 0$ as $|\mathrm{a}| \rightarrow 1^{-}$, where

$$
I(a)=\int_{\mathbb{D}}\left|f^{\prime}(z)\right|^{p} \frac{(1-|z|)^{q}}{\omega^{p}(1-|z|)} K\left(\log \frac{1}{|z|}\right) d A(z) .
$$


From the proof of Theorem 3.2, we know that $f \in Q_{\kappa, \omega}(p, q)$ implies that

$$
\int_{0}^{1}\left(\sum_{k=1}^{\infty} n_{k}\left|a_{k}\right| r^{\left(n_{k}-1\right)}\right)^{p} \frac{(1-r)^{q}}{\omega^{p}\left(\log \frac{1}{r}\right)} K\left(\log \frac{1}{r}\right) r d r<\infty .
$$

For any $\epsilon<0$, there is a $\delta \in(0,1)$ such that

$$
\int_{\delta}^{1}\left(\sum_{k=1}^{\infty} n_{k}\left|a_{k}\right| r^{\left(n_{k}-1\right)}\right)^{p}(1-r)^{q} \frac{K\left(\log \frac{1}{r}\right)}{\omega^{p}\left(\log \frac{1}{r}\right)} d r<\epsilon .
$$

We may as well assume that $\lim _{|\mathbf{a}| \rightarrow 1^{-}} K\left(\log \frac{1}{|\mathbf{a}|}\right)=0$. If $K$ satisfies the condition (11). Then we choose a such that $1>|\mathrm{a}|>\delta$. By Lemma 2.6. Theorem 2.7 and Theorem 3.2, we have

$$
\begin{aligned}
& \int_{0}^{\delta}\left(\sum_{k=1}^{\infty} n_{k}\left|a_{k}\right| r^{\left(n_{k}-1\right)}\right)^{p} \frac{(1-r)^{q}}{\omega^{p}(1-r)} \int_{0}^{2 \pi} K(g(z, a)) r d r d \theta \\
& \leq \int_{0}^{\delta}\left(\sum_{k=1}^{\infty} n_{k}\left|a_{k}\right| r^{\left(n_{k}-1\right)}\right)^{p} \frac{(1-r)^{q}}{\omega^{p}(1-r)} K\left(\log \frac{1}{|a|}\right) d r \\
& =K\left(\log \frac{1}{|a|}\right) \int_{0}^{\delta}\left(\sum_{k=1}^{\infty} n_{k}\left|a_{k}\right| r^{\left(n_{k}-1\right)}\right)^{p} \frac{(1-r)^{q}}{\omega^{p}(1-r)} d r \\
& \leq \frac{K\left(\log \frac{1}{|a|}\right)}{K\left(\log \frac{1}{\delta}\right)} \int_{0}^{\delta}\left(\sum_{k=1}^{\infty} n_{k}\left|a_{k}\right| r^{\left(n_{k}-1\right)}\right)^{p}(1-r)^{q} \frac{K\left(\log \frac{1}{r}\right)}{\omega^{p}\left(\log \frac{1}{r}\right)} d r \\
& \leq \frac{K\left(\log \frac{1}{|a|}\right)}{K\left(\log \frac{1}{\delta}\right)} \sum_{k=1}^{\infty} n_{k}^{p-q-1}\left|a_{k}\right|^{p} \frac{K\left(\frac{1}{n_{k}}\right)}{\omega^{p}\left(\frac{1}{n_{k}}\right)} .
\end{aligned}
$$

Since $\epsilon$ is arbitrary, we conclude that $\mathrm{I}(\mathrm{a}) \rightarrow 0$ as $|\mathrm{a}| \rightarrow 1^{-}$. So $f \in \mathrm{Q}_{\kappa, w, 0}(\mathrm{p}, \mathrm{q})$ and the proof is complete.

Carefully checking the proof of Theorems 3.2 and 3.3 we also obtain the following sufficient condition for $f \in Q_{\kappa, \omega, 0}(p, q)$ and hence in $f \in Q_{K, \omega, 0}(p, q)$ in terms of Taylor coefficients.

Theorem 3.4. Let $0<p<\infty$ and $-1<\mathrm{q}<\infty$. Let $\mathrm{K}$ satisfy condition (1) and $\omega$ satisfy condition (2). Suppose that

$$
f(z)=\sum_{k=0}^{\infty} a_{k} z^{n_{k}},
$$

has Hadamard gaps, then $\mathrm{f}$ belongs to $\mathrm{Q}_{\mathrm{K}, \omega, \mathrm{O}}(\mathrm{p}, \mathrm{q})$ if and only if

$$
\sum_{k=0}^{\infty} n_{k}^{p-q-1}\left|a_{k}\right|^{p} \frac{K\left(\frac{1}{n_{k}}\right)}{\omega^{p}\left(\frac{1}{n_{k}}\right)}<\infty
$$

\section{Conclusion}

From Theorems 3.2, 3.3 and 3.4, we can give the following theorem; 
Theorem 4.1. Let $0<\mathrm{p}<\infty,-1<\mathrm{q}<\infty$. Let $\mathrm{K}$ satisfy condition (1) and $\omega$ satisfy condition (2). Suppose that

$$
f(z)=\sum_{k=0}^{\infty} a_{k} z^{n_{k}} \in H(\mathbb{D})
$$

has Hadamard gaps, then the following statements are equivalent:

(i) $f \in Q_{\kappa, \omega}(p, q)$;

(ii) $f \in Q_{\kappa, w, 0}(p, q)$;

(iii) $\sum_{k=0}^{\infty} n_{k}^{p-q-1}\left|a_{k}\right|^{p} \frac{K\left(\frac{1}{n_{k}}\right)}{\omega^{p}\left(\frac{1}{n_{k}}\right)}<\infty$.

Received: April 2013. Accepted: October 2013.

\section{References}

[1] K. L. Avetisyan, Sharp inclusions and lacunary series in mixed-norm spaces on the polydisc, Complex variables and elliptic equations, 58(2)(2013), 185195.

[2] A. Baernstein II, Analytic functions of bounded mean oscillation, in Aspects of Contemporary Complex Analysis, Academic Press, London, (1980), 3-36.

[3] J. S. Choa, A property of series of holomorphic homogeneous polynomials with Hadamard gaps, Bull. Aust. Math. Soc. 53(3)(1996), 479-484.

[4] A. El-Sayed Ahmed, Lacunary series in quaternion $\mathbf{B}^{\mathbf{p}, \mathbf{q}}$ spaces, Complex variables and elliptic equations, Vol 54(7)(2009), 705-723.

[5] A.El-Sayed Ahmed, Lacunary series in weighted hyperholomorphic $\mathbf{B}^{\mathbf{p}, \mathbf{q}}(\mathbf{G})$ spaces, Numerical Functional Analysis and Optimization, Vol 32(1)(2011), 41-58.

[6] A. El-Sayed Ahmed, Meromorphic functions with Hadamard gaps,to appear.

[7] A. El-Sayed Ahmed and A. Kamal, Characterizations by gap series in meromorphic $\mathrm{Q}_{\mathrm{p}}$ functions, to appear.

[8] A. El-Sayed Ahmed and A. Kamal, Series expansions in some analytic function spaces, submitted.

[9] M. Essén, H. Wulan, and J. Xiao, Several function theoretic characterizations of Möbius invariant $\mathrm{Q}_{\mathrm{K}}$ spaces, J. Funct. Anal. 230(2006), 78-115.

[10] O. Furdui, On a class of lacunary series in BMOA, J. Math. Anal. Appl. 342(2)(2008), 773779 . 
[11] J. Garnett, Bounded analytic functions, Academic Press, New York, (1981).

[12] K. Gürlebeck and H. R. Malonek, On strict inclusions of weighted Dirichlet spaces of monogenic functions, Bull. Aust. Math. Soc. 64(2001), 33-50.

[13] K. Gürlebeck and A. El-Sayed Ahmed, On series expansions of hyperholomorphic B ${ }^{\mathrm{q}}$ functions, in Tao Qian et al. (Eds), Trends in Mathematics Advances in Analysis and Geometry, (Basel/Switzerland : Birkhäuser Verlarg Publisher) (2004), 113-129.

[14] A. Kamal and A. El-Sayed Ahmed, A property of meromorphic functions with Hadamard gaps, Scientific Research and Essays, Vol 8(15)(2013), 633-639.

[15] M. Mateljević and M. Pavlović, $\mathrm{L}^{\mathrm{p}}$-behavior of power series with positive coefficients and Hardy spaces, Proc. Amer. Math. Soc, 87 (1983), 309 - 316.

[16] J. Miao, A property of analytic functions with Hadamard gaps, Bull. Austral. Math. Soc. 45(1992), 105-112.

[17] R. A. Rashwan, A. El-Sayed Ahmed and Alaa Kamal, Integral characterizations of weighted Bloch spaces and $\mathrm{Q}_{\mathrm{K}, \omega}(\mathrm{p}, \mathrm{q})$ spaces, Mathematica 51(74)(1)(2009), 63-76.

[18] W. Rudin, Real and complex analysis, MeGraw-Hill, New York, (1987).

[19] H. Wulan and K. Zhu, Lacunary series in $\mathrm{Q}_{\mathrm{K}}$ spaces, Studia Math, 178 (2007), 217-230.

[20] H. Wulan and K. Zhu,Lacunary series in $\mathrm{Q}_{\mathrm{K}}$ spaces, Journal of function spaces and applications, Vol 6(3)(2008), 293-301.

[21] A. Zygmund, Trigonometric series. Volumes I and II combined. 1. paperback ed. of the 2. ed. Cambridge Mathematical Library. Cambridge University Press. XIV, (1988). Trigonometric series, Cambridge (1968). 